\title{
A novel mutation in nuclear prelamin a recognition factor-like causes diffuse pulmonary arteriovenous malformations
}

\author{
Hong-zhou Liu ${ }^{1, *}$, Chun-xian Du ${ }^{2, *}$, Jing Luo ${ }^{1, *}$, Xue-ping Qiu ${ }^{1}$, Zu-hua Li ${ }^{1}$, Qi-yong \\ Lou $^{3}$, Zhan Yin ${ }^{3}$, Fang Zheng ${ }^{1}$ \\ ${ }^{1}$ Center for Gene Diagnosis, Zhongnan Hospital of Wuhan University, Wuhan, Hubei, 430071, China \\ ${ }^{2}$ Department of Pneumology, Zhongnan Hospital of Wuhan University, Wuhan, Hubei, 430071, China \\ ${ }^{3}$ Key Laboratory of Aquatic Biodiversity and Conservation of Chinese Academy of Sciences, Institute of Hydrobiology, Chinese \\ Academy of Sciences, Wuhan 430072, Hubei, China \\ *These authors have contributed equally to this work \\ Correspondence to: Fang Zheng, email: zhengfang@whu.edu.cn \\ Keywords: pulmonary arteriovenous malformations, capillary malformations, nuclear prelamin A recognition factor-like, whole \\ exome sequencing, zebrafish \\ Received: August 15, $2016 \quad$ Accepted: October 12, $2016 \quad$ Published: November 07, 2016
}

\section{ABSTRACT}

Two daughters in a Chinese consanguineous family were diagnosed as diffuse pulmonary arteriovenous malformations (PAVMs) and screened using whole exome sequencing (WES) and copy number variations (CNVs) chips. Though no mutation was found in the established causative genes of capillary malformation-AVMs (CM-AVMs) or PAVMs, Ser161Ile (hg19 NM_022493 c.482G $>$ T) mutation in nuclear prelamin A recognition factor-like (NARFL) was identified. Ser161Ile mutation in NARFL conservation region was predicted to be deleterious and absent in $\mathbf{5 0 0}$ population controls and Exome Aggregation Consortium (ExAC) Database. And there was a dosage effect of the mutation on mRNA levels among family members and population controls, consistent with the instability of mutant mRNA in vitro. Accordingly, in lung tissue of the proband, NARFL protein expression was reduced but $\mathrm{Fe}^{3+}$ was overloaded with vascular endothelial growth factor (VEGF) overexpression. Furthermore, NARFL-knockdown cell lines demonstrated decreased activity of cytosolic aconitase, while NARFL-knockout zebrafish presented ectopic subintestinal vessels sprouts and upregulated VEGF. So we concluded that the Ser161Ile mutant induced NARFL deficiency and eventually diffuse PAVMs probably through VEGF pathway. In a word, we detected a functional mutation in NARFL, which might be the pathogenic gene in this pedigree.

\section{INTRODUCTION}

Pulmonary arteriovenous malformations (PAVMs) have the latest incidence of $\sim 1$ in 2600 based on the diagnosis with the thoracic computed tomography $(\mathrm{CT})$ [1]. PAVMs are abnormal vessels diseases, as fistulous connections between pulmonary arteries and veins [2]. Diffuse PAVMs are recorded when PAVMs are involved in every subsegmental artery within at least one lobe [2-3]. Capillary malformations (CM), another kind of vessels disease, can be an isolated clinical disease or associated with syndromic vascular anomalies [4], such as Sturge-Weber, Klippel-Trenaunay, Parkes-Weber, CM-
AVMs [5-6], macrocephaly-CM (M-CM), and diffuse $\mathrm{CM}$ with overgrowth (DCMO) [3]. Among them, CMAVMs as capillary level PAVMs, are a newly recognized clinical entity [5-7]. So far, only Endoglin (ENG), activin A receptor type II-like 1 (ACVRL1), Mothers against decapentapledic homolog 4 (SMAD4), and RAS activator 1 (RASA1) were reported to be causal genes of PAVMs and CM-AVMs [7-11].

Here, we describe a family where two female offsprings of first cousin parents presenting diffuse PAVMs. To identify the causative mutations, whole exome sequencing (WES) and copy number variations (CNVs) chips were performed in the two daughters 
and the parents. And functional studies were carried on the identified possible causal gene nuclear prelamin $A$ recognition factor-like (NARFL).

NARFL was also known as iron-only hydrogenaselike protein 1 (IOP1) [12]. It was a ubiquitous iron-sulfur $(\mathrm{Fe}-\mathrm{S})$ protein in every living cell and played an essential role in $\mathrm{Fe}-\mathrm{S}$ protein biogenesis in many physiologic processes, including electron transport, enzyme catalysis, and Krebs cycle [13-14].

\section{RESULTS}

\section{Clinical diagnosis}

The 20-year-old proband was admitted to Center for Gene Diagnosis, Zhongnan Hospital of Wuhan University with cyanosis, dyspnea, clubbing, voice hoarse, and severe hyoxemia. Echocardiography revealed pulmonary arterial hypertension (PAH). Arterial blood gas analysis revealed right-to-left shunting ( $\mathrm{pH}=7.457, \mathrm{PaO}_{2}=348 \mathrm{mmHg}$ $(<500 \mathrm{mmHg}))$. Chest X-ray and computed tomography (CT) scan showed a ground glass appearance indicating inflammation (Figure 1A). Enhanced CT scan showed pulmonary arteriovenous malformation (Figure 1C). Lung biopsies showed diffuse PAVMs (Figure 1F, 1G, 1H, 1I). The proband first appeared dyspnea on exertion at 12-year-old. Then she had hemoptysis and was diagnosed as TB infection followed by anti-TB drugs treatment for seven months at 18-year-old. After the proband suffered from progressive dyspnea for one year, she was diagnosed as diffuse PAVMs at 20-year-old mainly based on lung biopsies. Eventually, she died of pulmonary hypertension and cardiac and respiratory failure despite treatment with $50 \mathrm{mg}$ steroids for one month at 22-year-old. The proband's elder sister also had occasional hemoptysis, dyspnea on exertion, clubbing and voice hoarse. She was diagnosed as diffuse PAVMs mainly based on imageology results (Figure 1B, 1D, 1E).

\section{Identification of novel mutations}

The entire list of candidate variants was listed in Supplementary Table S1 online and only 1737 proteinaltering variants were considered [15]. Then the variants were filtered against BGI-Shenzhen four inner databases, dbSNP137, HapMap8 and 1000 human genome databases and only 459 variants were considered. Filter variants were predicted by the SIFT software and 110 variants were considered because of damaging prediction. Among the 110 variants, 4 variants of genes which were ubiquitously expressed, such as in vessels and lungs according to the Uniprot. Only Ser161Ile (hg19 NM_022493 c.482G>T) in NARFL gene co-segregated with the phenotype in the family and was not present in Exome Aggregation Consortium (ExAC) Database (http://exac.broadinstitute. org). The mutation is a polar amino acid (Ser) replaced by the nonpolar (Ile) in the 161st residue of NARFL, which is highly conserved in evolution. The mutation was confirmed by Sanger sequencing (Figure 2A) and absent in 500 population controls. No candidate CNVs according to the autosomal recessive model were found and were reported to be associated with PAVMs and CM-AVMs (Supplementary Table S2 online). Furthermore, NARFL gene of thirty five sporadic patients with similar symptoms was sequenced and one new polymorphism of Leu299Met (hg19 NM_022493 c.895C >A) was found.

\section{In silico prediction of mutant NARFL proteins}

The Ser161Ile (hg19 NM_022493 c.482G>T) in NARFL was predicted to be deleterious using SIFT and Polyphen-2. Furthermore, in the Ser161Ile mutant, the distance of hydrogen bond between Ser161 (Ile161) and $\operatorname{Arg} 158$ increased from 3.20 to 3.22 (Figure 2B) in the cavity of NARFL protein (Figure 2D). However, no change of the helix at position 161 was observed (Figure 2C). And the hydropathicity and hydrophobicity of mutant NARFL (Ser161Ile) were affected slightly (Figure 2E). Considering that in silico prediction of the structure change of mutant proteins was slight, we hypothesized this novel mutation Ser161Ile might affect the NARFL mRNA level.

\section{Decreased level and stability of the mutant NARFL mRNA}

There was a decrease in mutant NARFL mRNA levels in vivo (Figure $3 \mathrm{~A}$ ). The sisters were homozygotes and their relative expression levels of NARFL mRNA decreased by 0.36 -fold, while the parents and the brother were heterozygotes with relative expression levels of 0.51 fold, compared with population controls $(p<0.01)$.

Sequentially, as shown in vitro, mRNA levels of Ser161Ile mutant ( $\mathrm{t}_{\text {hal-time }}=20$ hours $)$ dropped significantly in transfected cells, compared with the wild-type ( $\mathrm{t}_{\text {half- }}$ $=34$ hours) $(p<0.05, n=3$, Figure 3B). And this phenomenon was also observed in protein levels (Figure 3C, 3D). These indicated that NARFL mRNA levels were declined in mutation carriers probably because of mRNA instability. Accordingly, the NARFL protein expression in the proband's lung tissue was significantly reduced compared with controls ( $p=0.002, n=6$, Figure 3E, 3F). In addition, the $\mathrm{Fe}^{3+}$ overload and vascular endothelial growth factor (VEGF) overexpression were both observed in the proband's lung tissue compared with controls $(p=$ $0.0009, n=6 \& p=0.0001, n=6$, Figure 3G, 3H, 3I, 3J).

\section{NARFL knockdown induced declined cytosolic $\mathrm{Fe}-\mathrm{S}$ protein aconitase activity in cells}

Among the five synthesized short hairpin RNA (shRNA) duplexes, the shRNA-A and shRNA-B knocked down NARFL expression significantly in HEK293T cells 
$(p<0.01, n=3$, Figure 4A, 4B, 4C). We found NARFL knockdown reduced activity of cytosolic aconitase, a Fe-S protein, significantly (both $p<0.01, n=6$, Figure 4D), but not mitochondrial aconitase and lactate dehydrogenase (LDH) activity. These indicated that NARFL knockdown might affect its function of transferring Fe-S cluster and further biogenesis of cytosolic $\mathrm{Fe}-\mathrm{S}$ proteins, including aconitase in cell lines. These could be the reason for $\mathrm{Fe}^{3+}$ overload in the proband's lung.

\section{NARFL knockout led to aberrant angiogenesis in zebrafish}

NARFL knockout experiments in zebrafish were performed to identify whether NARFL knockout would exhibit a vascular phenotype. As shown in Figure 4F, most of control fish at 3 days postfertilization $(\mathrm{dpf})(\mathrm{n}=94 / 126)$ exhibited an orderly subintestinal vessels. However, NARFL knockout zebrafish presented ectopic subintestinal vessels sprouts (Figure 4F) $(p<0.001, n=22 / 109$ versus 94/126), and VEGF overexpression was also detected (Figure 4G). As shown in Figure 4G, VEGF mRNA levels of NARFL (-/-) zebrafish were significantly higher than the wild type $(p<0.01)$. In addition, the expression of superoxide dismutase (SOD) and catalase (CAT) was both declined $(p<0.01)$.

\section{DISCUSSION}

We identified a probable causative gene $N A R F L$ (Ser161Ile) in a family with diffuse PAVMs, and the diffuse PAVMs were further demonstrated by positive
A

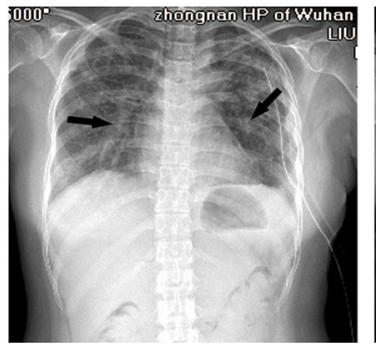

B

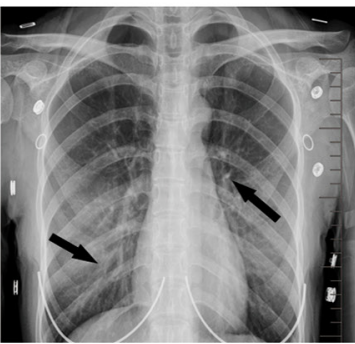

C

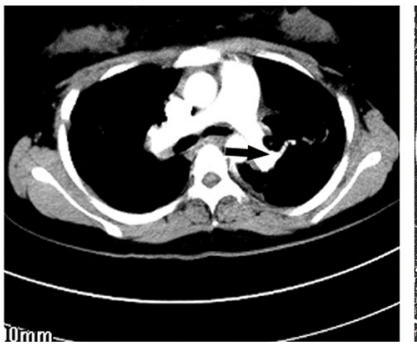

$\mathbf{G}$

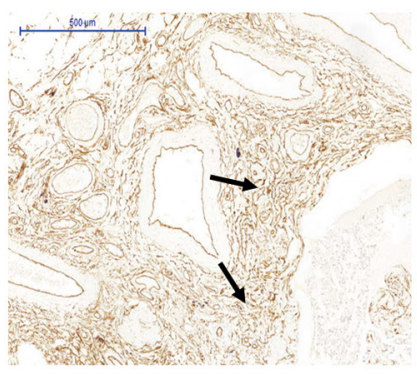

D

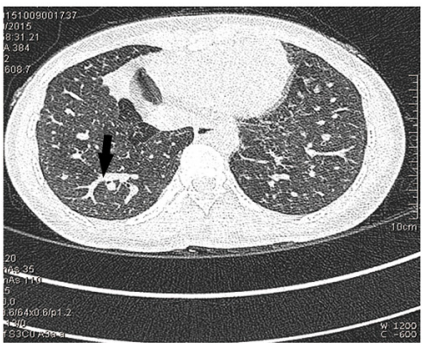

$\mathbf{E}$

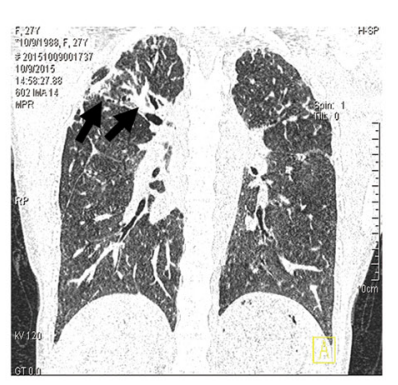

$\mathbf{F}$

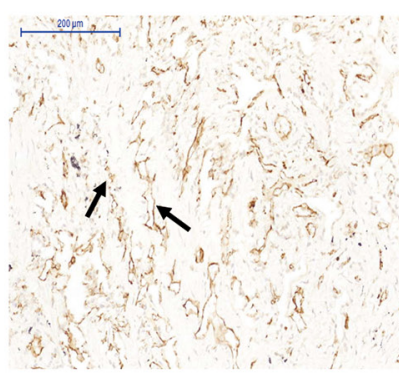

H

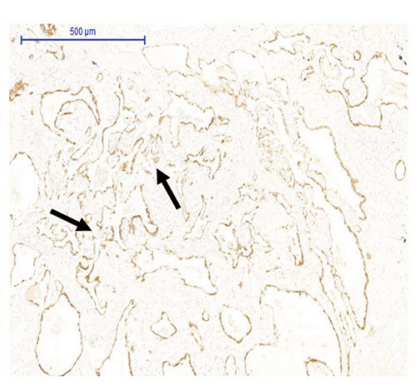

I

$\mathbf{J}$
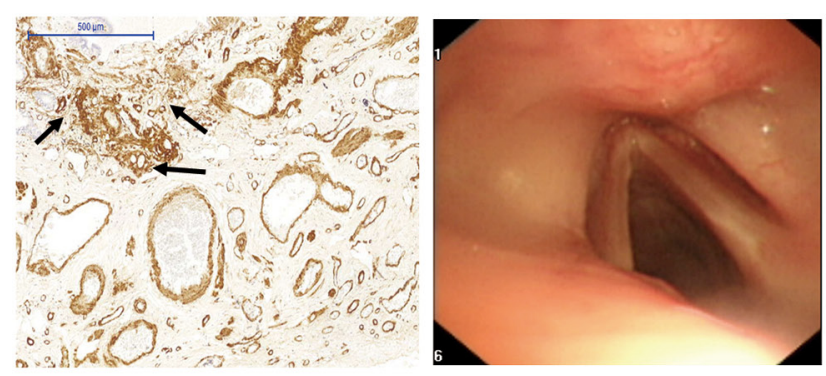

Figure 1: Clinical characteristics of patients. A, B. X-ray showed ground glass appearance (arrows) in lungs of the proband (A) and the sister (B). C. Enhanced CT scans showed AVMs in lungs of the proband. D, E. CT scans showed AVMs and diffuse pulmonary interstitial inflammation (arrows) in the proband (D) and the sister (E). F. Immunostaining for CD31 showed diffuse vascular malformation (arrows) without any recognizable architectural structure in lung biopsies of the proband $(\times 100)$. G, H. and I. Immunostaining for CD34 $(\mathrm{G})$, SMA $(\mathrm{H})$, and CK (I) showed diffuse vascular malformation (arrows) without any recognizable architectural structure in lung biopsies of the proband $(\times 50)$. J. Fiberoptic bronchoscopy showed no telangiectases or other dermal lesions. 
immunostaining for platelet endothelial cell adhesion molecule-1 (CD31), CD34, cytokeratin (CK), and a-smooth muscle actin (SMA) [16]. The Ser161Ile mutation caused the mRNA instability and reduced the NARFL mutant's expression in vivo. NARFL knockdown

A

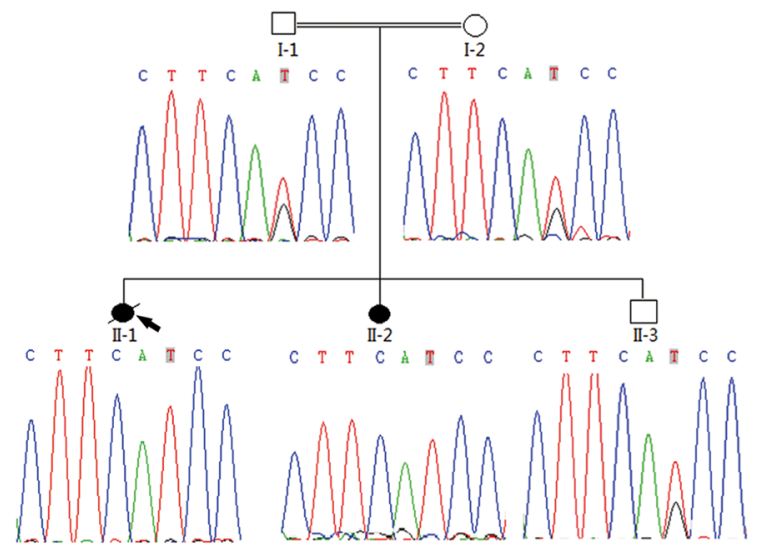

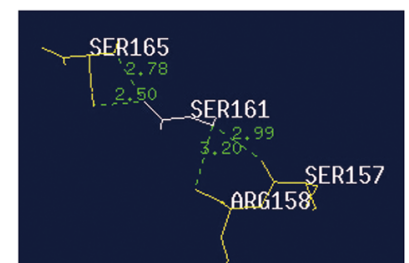

wild-type

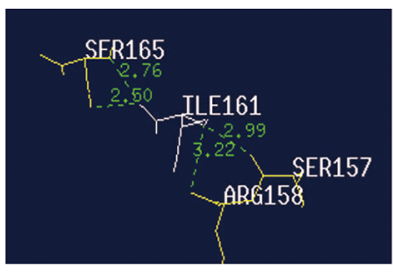

mutant-type

C

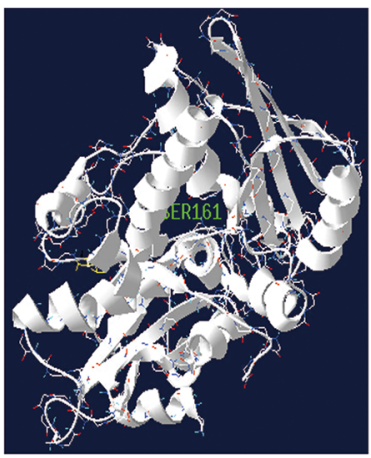

wild-type

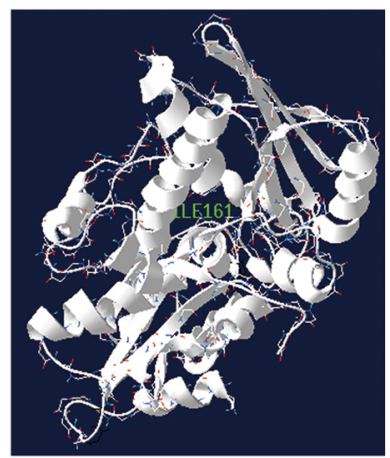

mutant-type

D

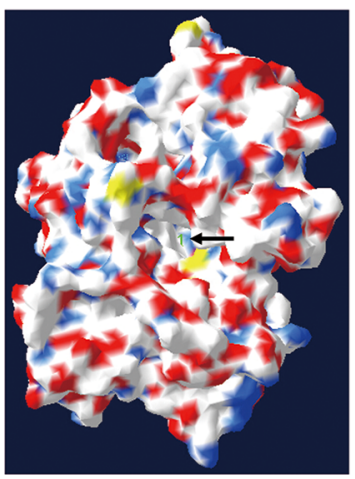

wild-type

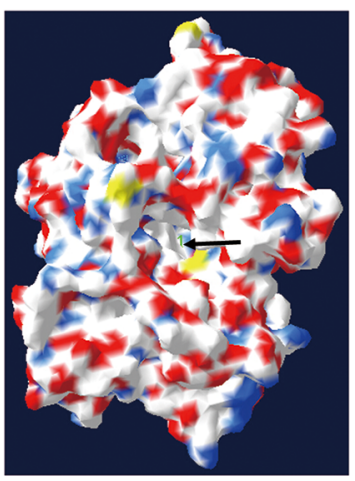

mutant-type

$\mathbf{E}$
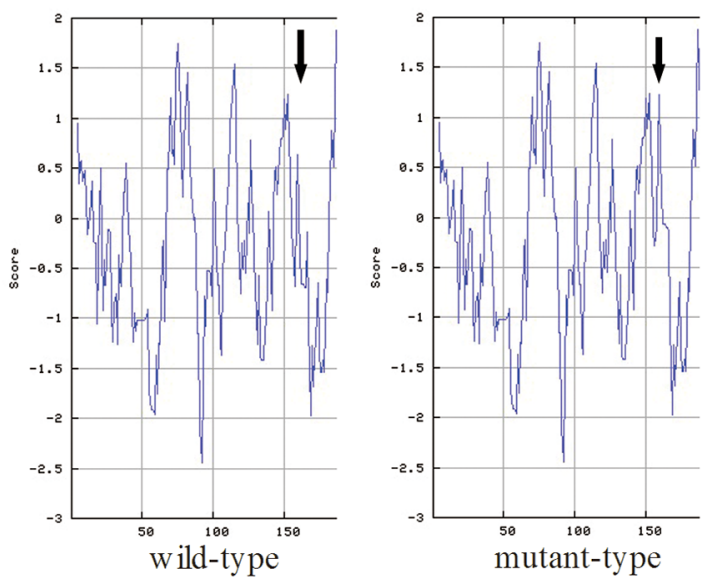

Figure 2: In silico prediction of mutant $N \boldsymbol{A R F L}$. A. Sequence chromatograms showed the mutation Ser161Ile in NARFL of the family members. B. The distance of hydrogen bonds in the NARFL (Ser161Ile) was increased (from 3.20 to 3.22). C. The helix was unaffected in the secondary structure of the Ser161Ile. D. The Ser161 was in cavity (arrows) of NARFL in the tertiary structure, which might be active center. E. Hydropathicity and hydrophobicity of the Ser161Ile were affected. 
A

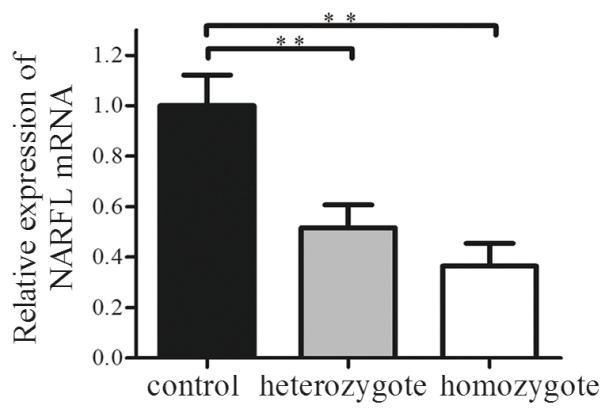

C

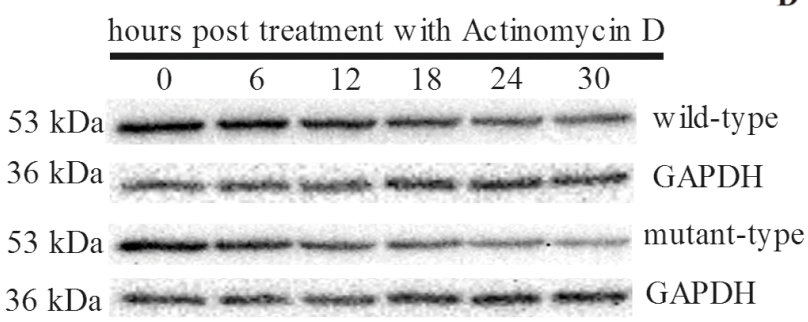

E

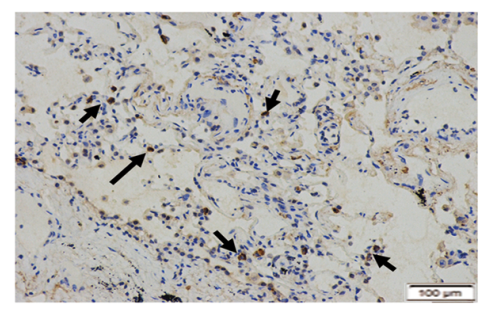

G

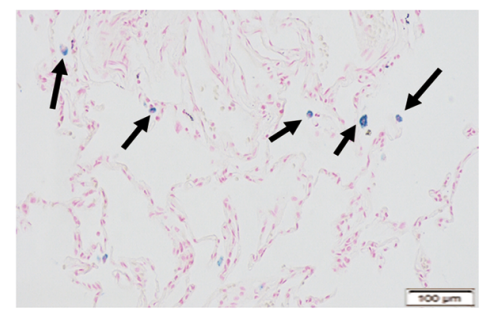

I

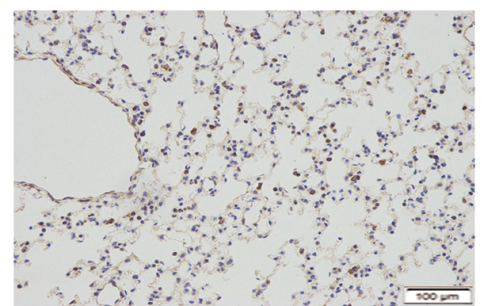

B

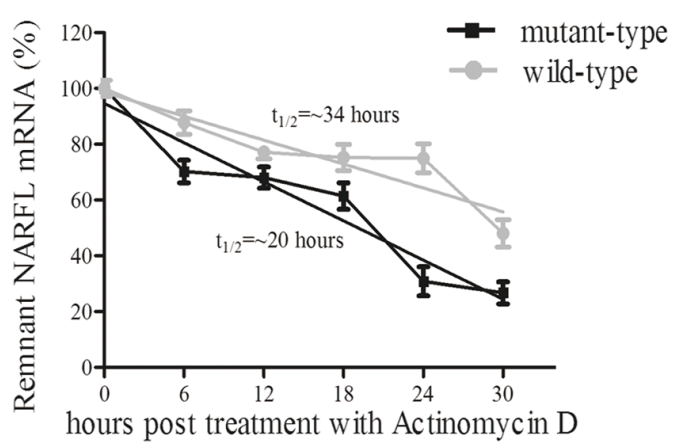

D

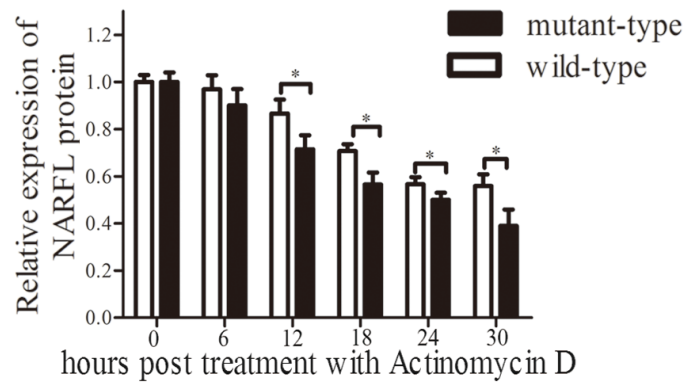

F

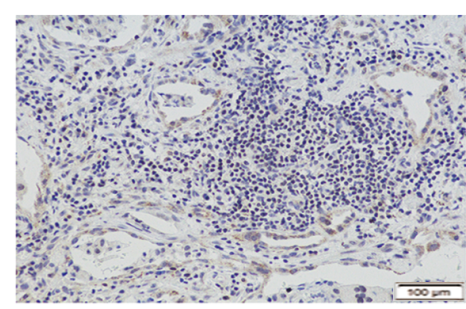

H

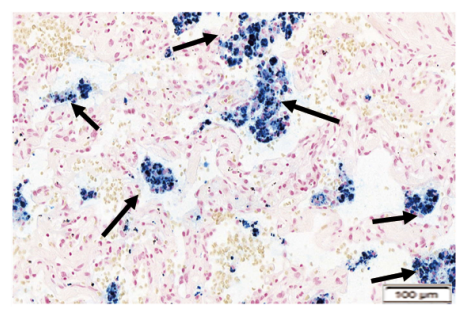

J

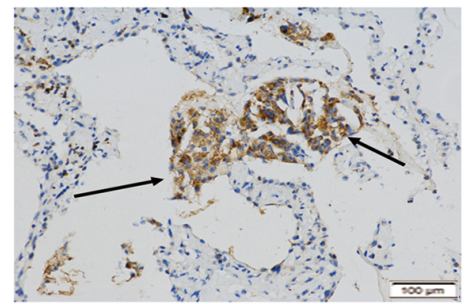

Figure 3: NARFL Expression in family members and results of mRNA stability assay. A. NARFL mRNA levels in heterozygote $(\mathrm{n}=3)$ and homozygote $(\mathrm{n}=2)$ were significantly lower than population controls $(\mathrm{n}=6)$. B. Mutant NARFL mRNA level decreased more sharply than wild type $N A R F L$ after actinomycin D treatment. The $N A R F L / G A P D H$ ratio was expressed as a percent of the value obtained for 0 hour post-treatment and then plotted as the semi-log to reflect the difference of mRNA decay rate. C. Mutant NARFL protein levels decreased more sharply than wild-type NARFL after actinomycin D treatment. D. NARFL protein levels were recorded as gray scale values and analyzed using Image J software. E, F. NARFL was examined using immunohistochemical staining, and strong expression of NARFL (indicated by arrows) was observed in the lung tissues of the controls (E) compared to the proband (F) $(\mathrm{n}=6, p=0.002)$. G, H. Lower level of $\mathrm{Fe}^{3+}$ (indicated by arrows) was observed in controls $(\mathrm{G})$ compared to the proband $(\mathrm{H})(\mathrm{n}=6, p=0.0009)$. I, J. Lower expression level of VEGF (indicated by arrows) was observed in controls (I) compared to the proband ( $\mathrm{J})(\mathrm{n}=6, p=0.0001)$. Images E-J are $\times 200$ magnification. $\left({ }^{*}, p<0.05 ;{ }^{* *}, p<0.01\right)$ 
Firstly, we found that Ser161Ile mutant only induced slight distance changes of hydrogen bonds in cavity of NARFL protein, and slightly influenced the hydropathicity and hydrophobicity. However, we observed there was a dosage effect of Ser161Ile mutant on NARFL mRNA expression, though only a decreased tendency of mRNA levels occurred among family members. This might be due to the limited sample size or other confounding factors.
Secondly, NARFL deficiency further reduced the activity of cytosolic aconitase instead of mitochondrial aconitase in NARFL-knockdown cell lines. This was understandable, since NARFL is reported to be a unique component in cytosolic iron-sulfur cluster assembly (CIA) system and functions mainly in biogenesis of cytosolic iron-sulfur protein [14]. NARFL serves as a scaffold for delivering iron-sulfur cluster to iron regulatory protein 1

COS7 HUVEC HEK293T Hep3B

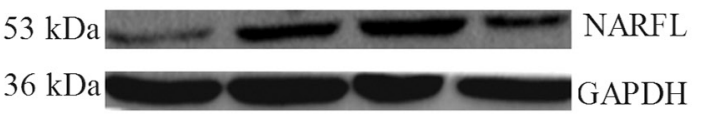

C

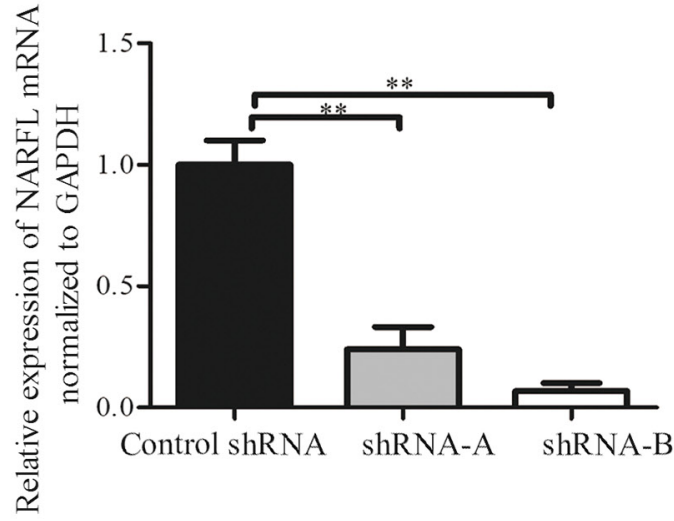

E

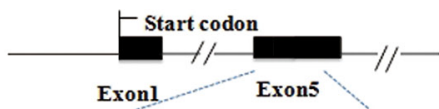

wild-type GGCTITACCGATGCTGGCATCTGCATGTCCAGGTAGCCTC

mutant-type GGCTTTACCGAT TIACGTTGGCATCTGCATGTCCAGGTA

G

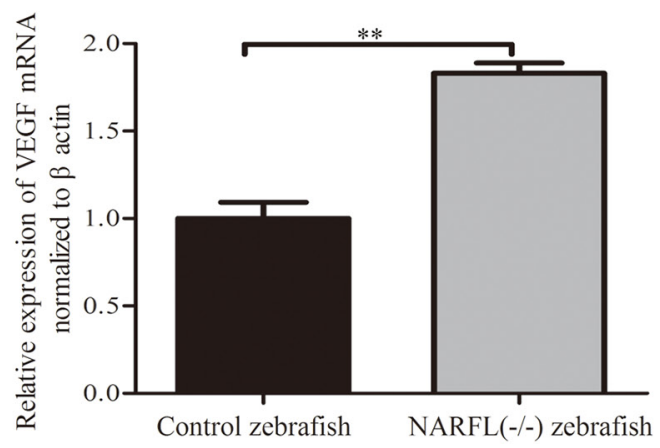

B
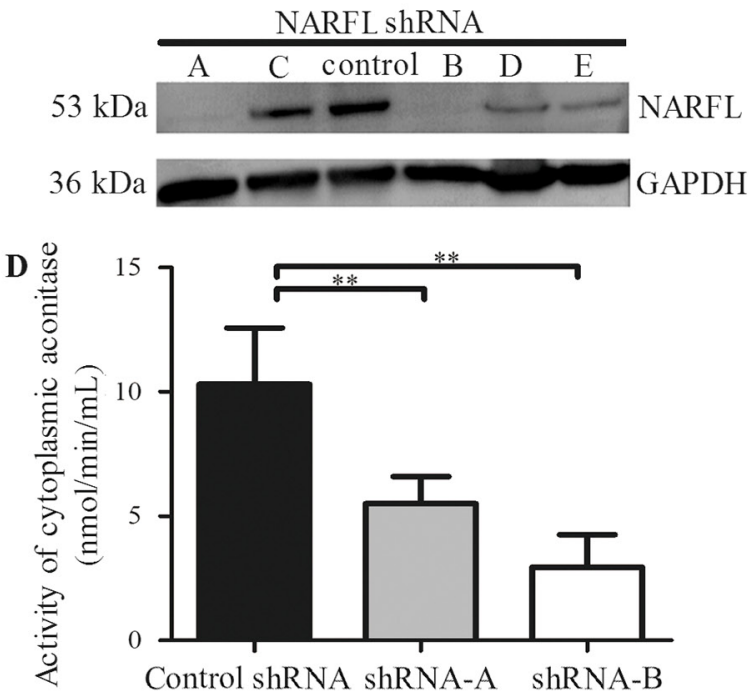

$\mathbf{F}$
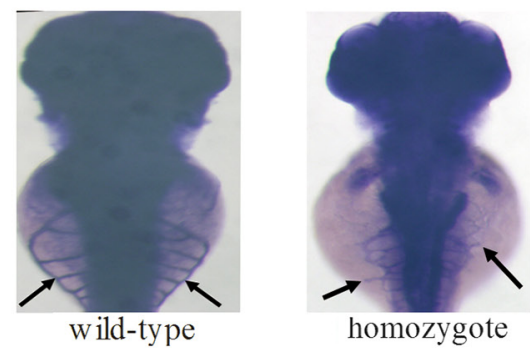

H

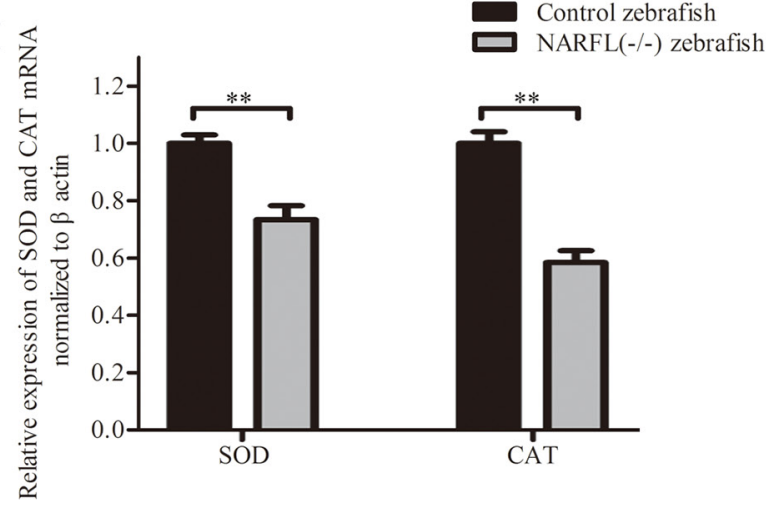

Figure 4: Effects of NARFL-knockdown in HEK293T cells and NARFL-knockout in zebrafish. A. NARFL was highly expressed in HEK293T cells detected using western blot. B, C, D. shRNA-A and shRNA-B of five shRNA duplexes (B) induced declined NARFL mRNA level (C) and reduced aconitase activity (D) in HEK293T cells. E. Targeted indel mutations induced by CRISPR/Cas9 at the NARFL gene. The wild-type sequence was shown at the top with the target sites highlighted as blue and the mutant sequence highlighted as red. F. Control zebrafish (94/126) showed dominant subintestinal vessels in 3 dpfs using blood vessel staining. In NARFL knockout zebrafish (87/109), the subintestinal plexus has ectopic sprouts $(p<0.0001, n=22 / 109$ versus 94/126). G. VEGF mRNA levels were examined using qRT-PCR assay and VEGF mRNA levels were upregulated in NARFL-knockout zebrafish. H.The mRNA levels of SOD and CAT were declined. $(*, p<0.05 ; * *, p<0.01)$ 
(IRP1), to form cytosolic aconitase which is an important Fe-S protein $[12,14,18]$. So the deficiency of NARFL might impair the biogenesis of cytosolic Fe-S proteins and lead to inactivation of cytosolic aconitase. Furthermore, NARFL knockdown was reported to upregulate transferrin receptor 1 (involved in iron uptake) which could directly increase intracellular iron amount [12]. And cytosolic aconitase, upon loss of its iron-sulfur cluster, converts to IRP1, which could bind the 5'-untranslated regions of the ferritin heavy chain mRNA. IRP1 in turn serves to inhibit translation of ferritin heavy chain protein which is an intracellular iron storage protein, and further increases free iron levels in cells [18-19]. So the deficiency of NARFL and inactivation of cytosolic aconitase could both lead to iron uptake and storage impairment in lung tissue of the proband (as shown in Figure $3 \mathrm{H}$ ).

Thirdly, iron overload could directly promote VEGF expression [20], which could explain the iron overload and VEGF overexpression in the proband (as shown in Figure 3J). VEGF overexpression further led to aberrant vascularization, which was confirmed by the ectopic subintestinal vessels sprouts and VEGF overexpression in NARFL(-/-) zebrafish.

In another aspect, the pathogenesis might be abnormally increased oxidative stress. NARFL was recently reported to be a key component to defend oxidative stress, companying with loss of cytosolic aconitase activity [13]. Decreased aconitase activity could in turn lead to oxidative stress through iron overload [17-18]. Iron overload activates proteins oxidation in the Fenton reaction, leading to $\mathrm{Fe}^{3+}$ generation and accumulation, which could be demonstrated by Prussian blue staining as shown in Figure $3 \mathrm{H}$ as previously reported [19]. So far, aconitase activity is widely used as a biomarker for oxidative stress, as high-intensity exercises in humans lead to inactivation of aconitase due to ROS generation [19, 21-23]. These indicated that functional aconitase was essential in maintaining redox balance and the cellular antioxidant defense system [22]. Therefore, we inferred that deficiency of NARFL promoted production of ROS, either directly [13] or via decreased aconitase activity, resulted in iron overload [19-25]. And many studies had demonstrated that increased ROS initiated pathological angiogenesis and vascular malformations via VEGF signaling pathway [26-27]. These could also be the reason for the abnormal angiogenesis in NARFL-knockout fish and the proband's lung tissue.

\section{Limitations}

Only one family with $N A R F L$ mutations was found, though the cases were really rare. The complex interactions among genes were not considered in this consanguineous family. Some phenotypes except diffuse PAVMs such as interstitial pneumonitis required to be explained in the future [28].
In a word, we have identified NARFL as a possible genetic basis underlying autosomal recessive diffuse PAVMs.

\section{MATERIALS AND METHODS}

\section{Patients and ethics}

A consanguineous family having two daughters both diagnosed as diffuse PAVMs were recruited in Center for Gene Diagnosis, Zhongnan Hospital of Wuhan University. Investigation has been conducted according to the Declaration of Helsinki and approved by the Ethics committee of Zhongnan Hospital of Wuhan University. After informed consent was obtained from all 5 family members, 35 sporadic patients and 500 population controls, DNA and total RNA were extracted from white blood cells using the method as previous [29].

\section{Whole exome sequencing}

WES was performed on two daughters and their parents at BGI-Shenzhen (Shenzhen, China). Exome enrichment was performed with Agilent Sure Select Human All Exon v2 kit (50Mb) (Agilent Technologies, Inc. SantaClara, CA) with an average sequencing depth of 70 -fold and coverage of $97.7 \%$. Enriched shotgun libraries were sequenced with the Illumina Hiseq2000 platform (Illumina, Inc., San Diego, CA). Raw image data and base calling were processed by Illumina Pipeline software v1.7 and SOAP aligner was used to align the high quality reads to the human reference genome (hg19). A list of qualifying genotypes was generated by filter criteria [30]. First, only protein-altering variants, such as missense variants, frame shift, indels and intron-exon boundary variants were included. Second, the variants were absent in the available four BGI-Shenzhen inhouse databases. Third, the variants were also absent in the dbSNP137, HapMap, 1000 human genome dataset. Fourth, autosomal recessive models were systematically considered. Fifth, qualifying genes were checked against Uniprot (http://www.uniprot.org/) to be filtered whether it was expressed in lung or ubiquitously expressed. Sixth, ExAC (http://exac.broadinstitute.org) was used to assess the variant's frequency and influence.

\section{Comparative genomic hybridization}

Comparative genomic hybridization ( $\mathrm{CGH}$ ) on daughters and their parents were performed in CapitalBio Cooperation (Beijing, China) to discover large deletions and copy number variations (CNVs). Human $\mathrm{CGH}$ $3 \times 1.4 \mathrm{M}$ Whole-Genome Exon-Focused Array (Roche Diagnostics, Mannheim, Germany) was used with a median spacing of $7 \mathrm{~kb}$ and 5 probes/exon. Raw CGH data were extracted and analyzed using Nimble Scan v2.6 
software (Roche Diagnostics) and CNVs were identified by filtering more than 5 consecutive probe segments with $\left[\log _{2}\right.$ ratio $]>0.25$.

\section{Sanger sequencing of the variants}

All validated variants were subsequently verified in 5 family members by Sanger sequencing. Furthermore, the mutation in NARFL gene was screened in 35 sporadic patients and 500 population controls using single strand conformation polymorphism (SSCP) analysis and confirmed using Sanger sequencing.

\section{In silico prediction of NARFL protein}

SIFT (http://sift.jcvi.org/) and Polyphen-2 (http:// genetics.bwh.harvard.edu/pph2) were used for assessing biological effects of the mutation. Multiple NARFL protein sequence alignments across species were performed using ClustalW2. SWISS-Model modeling server (http://swissmodel.expasy.org/) and ExPASy (http:// www.expasy.org/tools/protparam) were used to analyze the protein structure, conserved domain and functional domain.

\section{Quantitative real time reverse transcript PCR}

cDNA was synthesized using the Revert Aid First Strand cDNA Synthsis Kit (Fermentas, Burlington, Ontario, Canada). The expression levels of NARFL were detected by quantitative real time reverse transcript PCR (qRT-PCR) in a CFX 96 real time system (Bio-Rad, Shanghai, China) using SYBR mix (Applied Biosystem, Shanghai, China). The human samples were assayed in triplicate and glyceraldehydes-3-phosphate dehydrogenase (GAPDH) was used as an internal control. The mRNA expression levels of VEGF were compared between NARFL (-/-) zebrafish and controls (30 zebrafish per group) using qRT-PCR as described above. The zebrafish samples were assayed in triplicate and $\beta$-actin was used as a control. Relative gene expression levels were determined by the $2^{-\Delta \Delta C t}$ method.

\section{Cell lines, plasmids and transfection}

HEK293T, COS7, Hep3B cell lines were obtained from American Type Culture Collection (ATCC, Manassas, VA). Human umbilical vein endothelial cells (HUVECs) were obtained from Cell Bank of Shanghai Institutes for Biological Sciences. HEK293T cells were grown in Dulbecco's modified Eagle's medium and transfected with lipofectamine 2000 (Invitrogen, Shanghai, China). The pCMV-HA-wild plasmid was constructed by amplifying controls' cDNA and the pCMV-HA-mutant plasmid was prepared using a QuikChange mutagenesis kit (Stratagene, Shanghai, China).

\section{The mRNA stability assay}

The pCMV-HA-wild and pCMV-HA-mutant were transfected into HEK293T cells, respectively. The cells were harvested and total RNA was isolated once Actinomycin D (Sigma, USA) was added to inhibit RNA synthesis, at the indicated hours. Then NARFL mRNA level was quantified using qRT-PCR. The experiment was repeated for three times and the mean half-life of mRNA was compared by $t$ test.

\section{Western blotting}

Cellular protein samples were isolated using RIPAlysis buffer (Beyotime, Shanghai, China) and the protein concentration was measured by $\mathrm{BCA}$ assay (Beyotime). Antibodies to HA and Myc were obtained from Cell Signaling Technologies (GST, Danvers, USA). Antibody to NARFL was obtained from Abcam (Abcam, Shanghai, China). The membranes were incubated with HRP-conjugated secondary antibodies and the signals were visualized with ECL (Beyotime, Shanghai, China). The band densities of the proteins were measured with Image J software. GAPDH was selected as an internal control and the experiments were performed in triplicate.

\section{NARFL knockdown and enzyme assays}

The pSUPER-retro-control plasmid and five shRNA duplexes were synthesized in Invitrogen (Invitrogen, Shanghai, China). Cell mitochondria isolation kit was used for the separation of mitochondria and cytosol (Beyotime), and cytochrome $\mathrm{C}$ was detected using western blot to guarantee no cytosolic contamination in the mitochondrial component. Aconitase activity was measured by aconitase assay kit (Sigma, Shanghai, China) in mitochondria and cytosol, as well as lactate dehydrogenase activity using LDH assay kit (Beyotime).

\section{Immunohistochemical staining}

Lung tissues were collected from the proband and the population controls and were fixed in $10 \%$ buffered formalin. Antigen Unmasking Solution (Vector laboratories, QLD, Australia) was used for antigen retrieval and $10 \%$ horse serum was used for blocking. Sections of lung tissues were then incubated with NARFL, VEGF, CD31, CD34, SMA, and CK antibody (Abcam, Shanghai, China). The slides were then treated with secondary antibodies. Nuclei were visualized using Haematoxilin. The $\mathrm{Fe}^{3+}$ levels were detected using Prussian blue staining [31].

Expressions of NARFL and VEGF in lung tissues were determined using immunohistochemistry to assess the localization, intensity, and area of stained cells [32]. Intensity of the staining was graded as follows: no staining $=0$, mild staining $=1$, moderate staining $=2$ and intense 
staining $=3$. The area of staining was scored on the following scales: no stained cells in any microscopic field $=0,<25 \%$ of lung cells stained positively $=1,25 \%-50 \%$ $=2,50 \%-75 \%=3$ and $>75 \%=4$. The sum of the scores for area and intensity of staining were used for statistical analysis. At least six fields were randomly collected from each slide and were analyzed by Image $\mathrm{J}$ software.

\section{Zebrafish model}

All experiments involving zebrafish were performed in accordance with Chinese Legislation for Animal Experimentation and European Communities Council Directive 86/609/EEC. When required for observing phenotypes of live embryosno later than 5-dpf, embryos were anaesthetized by the addition of tricaine methanesulfonate to the embryo medium to a concentration of $150 \mathrm{mg} / \mathrm{L}$. Narfl(zebrafish)-knockout experiments were performed using clustered regularly interspaced short palindromic repeat (CRISPR)/Cas9 genome editing technology in zebrafish as previous [33]. A guide RNA (gRNA) was generated as described [34]. gRNA scaffold was cloned into pMD 19-T vector (TakaRa; cat\# D102A). Double-stranded DNA for specific gRNA synthesis was PCR amplified (Transgen; cat\# AS211) using the primers: Forward: 5' - TAATACGACTCACTATAGGGACATGC AGACGCCAACATGTTTTAGAGCTAGAAATAGC-3', Reverse: 5'-AGCACCGACTCGGTGCCACT-3'. After gel extraction, gRNA was synthesized using T7 RNA Polymerase (NEB; cat\# M0251S). Subsequently, 100pg gRNA and 150pg Cas9 mRNA (SBI, CAS500A-1) were injected into one or two-cell stage zebrafish embryos. Following hatching, 10 20 embryos were collected for DNA extraction. The target gene region was amplified using the primers: Forward: 5'AGACTCAAGTCAGAAGCCCTA-3', Reverse: 5' - CG TTCTTATTTTGACAGCAGCAC-3'. Gene sequencing was used to detect and characterize the mutation of narfl. The remainder of the embryos were raised up to adults as F0 and mated with wild-type zebrafish to generate the F1 generation. The F1 adults were genotyped by DNA sequencing of the PCR products amplified from their caudal fin. F1 strains harboring the detected mutations were crossed to each other to obtain the F2 generation. Blood vessels of zebrafish were stained using alkaline phosphatase staining [33].

\section{Statistics}

Results were expressed as mean \pm standard deviation. One-way ANOVA (F-test) was carried out for comparing vascular phenotypes of zebrafish between wild and mutant types. Comparison between two groups was carried out using the Student's test (t-test) such as mRNA and protein levels. Correlations in mRNA stability were assessed using the Karl Pearson coefficient of correlation.
For all tests, a $p$ - value of $\leq 0.05$ with two-tailed was considered to be of statistical significance.

\section{ACKNOWLEDGMENTS}

We thank all family members for their participation in this study.

\section{FUNDING}

Supported by the National Basic Research Program of China (973 Program, 2012CB720600) and the Program of National Natural Science Foundation of China (81071420/H2005).

\section{COMPETING FINANCIAL INTERESTS STATEMENT}

The authors declare no competing financial interests.

\section{Author contributions}

H.L. and F.Z. designed the experiments; C.D. collected the clinic data and performed the immunostaining; J.L., Q.L. and Z.Y. designed and performed the experiments of zebrafish; H.L., X.Q. and Z.L. performed the rest investigations and experiments; H.L., C.D. and F.Z. analyzed the data; F.Z. and Z.Y. provided technical and material support. F.Z. and H.L. wrote the manuscript; all authors reviewed the manuscript.

\section{REFERENCES}

1. Shovlin CL. Pulmonary arteriovenous malformations. Am J Respir Crit Care Med. 2014; 190: 1217-1228.

2. Kim HJ, Lee JS, Oh YM, Shim TS, Lim CM, Koh YS, Kim WS and Lee SD. Clinical characteristics of pulmonary arteriovenous malformations in Koreans. Respirology. 2015; 20: 155-159.

3. Rork JF, Alomari AI, Mulliken JB, Fishman SJ and Liang MG. Diffuse capillary malformation in association with fetal pleural effusion: report of five patients. Pediatr Dermatol. 2015; 32:70-75.

4. Garzon MC, Huang JT, Enjolras O and Frieden IJ. Vascular malformations. Part II: associated syndromes. J Am Acad Dermatol. 2007; 56:541-564.

5. Macmurdo CF, Wooderchak-Donahue W, Bayrak-Toydemir P, Le J, Wallenstein MB, Milla C, Teng JM, Bernstein JA and Stevenson DA. RASA1 somatic mutation and variable expressivity in capillary malformation/arteriovenous malformation (CM/AVM) syndrome. Am J Med Genet A. 2016; 170: 1450-1454.

6. Lubeck BA, Lapinski PE, Bauler TJ, Oliver JA, Hughes ED, Saunders TL and King PD. Blood vascular abnormalities 
in Rasa1 (R780Q) knockin mice: implications for the pathogenesis of capillary malformation-arteriovenous malformation. Am J Pathol. 2014; 184: 3163-3169.

7. Durrington HJ, Firth HV, Patient C, Belham M, Jayne D, Burrows N, Morrell NW and Chilvers ER. A novel RASA1 mutation causing capillary malformation-arteriovenous malformation (CM-AVM) presenting during pregnancy. Am J Med Genet Part A. 2013; 161A: 1690-1694.

8. Mallet C, Lamribet K, Giraud S, Dupuis-Girod S, Feige JJ, Bailly S and Tillet E. Functional analysis of endoglin mutations from hereditary hemorrhagic telangiectasia type 1 patients reveals different mechanisms for endoglin loss of function. Hum Mol Genet. 2015; 24:1142-1154.

9. Han C, Choe SW, Kim YH, Acharya AP, Keselowsky BG, Sorg BS, Lee YJ and Oh SP. VEGF neutralization can prevent and normalize arteriovenous malformations in an animal model for hereditary hemorrhagic telangiectasia 2. Angiogenesis. 2014; 17: 823-830.

10. Jelsig AM, Tørring PM, Kjeldsen AD, Qvist N, Bojesen A, Jensen UB, Andersen MK, Gerdes AM, Brusgaard K and Ousager LB. JP-HHT phenotype in Danish patients with SMAD4 mutations. Clin Genet. 2016; 90: 55-62.

11. Komiyama M, Ishiguro $T$, Yamada $O$, Morisaki $H$ and Morisaki T. Hereditary hemorrhagic telangiectasia in Japanese patients. J Hum Genet. 2014; 59: 37-41.

12. Song D and Lee FS. A role for IOP1 in mammalian cytosolic iron-sulfur protein biogenesis. J Biol Chem. 2008; 284: 9231-9238.

13. Corbin MV, Rockx DA, Oostra AB, Joenje $H$ and Dorsman JC. The iron-sulfur cluster assembly network component NARFL is a key element in the cellular defense against oxidative stress. Free Radic Biol Med. 2015; 89: 863-872.

14. Song $\mathrm{D}, \mathrm{Tu} \mathrm{Z}$ and Lee FS. Human ISCA1 interacts with IOP1/NARFL and functions in both cytosolic and mitochondrial Iron-Sulfur protein biogenesis. J Biol Chem. 2009; 284: 35297-35307.

15. Williams HJ, Hurst JR, Ocaka L, James C, Pao C, Chanudet E, Lescai F, Stanescu HC, Kleta R; GOSgene, Rosser $\mathrm{E}$, Bacchelli $\mathrm{C}$ and Beales P. The use of whole-exome sequencing to disentangle complex phenotypes. Eur J Hum Genet. 2016; 24: 298-301.

16. Lee SJ, Kim HY, Kim BS, Kim DW, Chung HY, Lee JM, Huh S and Bae HI. Capillary malformation of port-wine stain: differentiation from early arteriovenous malformation by histopathological clues. Am J Dermatopathol. 2012; 34: 523-528.

17. Sheftel A, Stehling $O$ and Lill R. Iron-sulfur proteins in health and disease. Trends Endocrinol and Metab. 2010; 21: 302-314.

18. Sharma AK, Pallesen LJ, Spang RJ and Walden WE. Cytosolic iron-sulfur cluster assembly (CIA) system: factors, mechanism, and relevance to cellular iron regulation, J Biol Chem. 2010; 285: 26745-26751.
19. Lushchak OV, Piroddi M, Galli F and Lushchak VI. Aconitase post-translational modification as a key in linkage between Krebs cycle, iron homeostasis, redox signaling, and metabolism of reactive oxygen species. Redox Rep. 2014; 19: 8-15.

20. Arjunan P, Gnanaprakasam JP, Ananth S, Romej MA, Rajalakshmi VK, Prasad PD, Martin PM, Gurusamy M, Thangaraju M, Bhutia YD,Ganapathy V. Increased retinal expression of the pro-angiogenic receptor GPR91 via BMP6 in a mouse model of Juvenile Hemochromatosis. Invest Ophthalmol Vis Sci. 2016; 57:1612-1619.

21. Gardner PR, Nguyen DD and White CW. Aconitase is a sensitive and critical target of oxygen poisoning in cultured mammalian cells and in rat lungs. Proc Natl Acad Sci USA. 1994; 91: 12248-12252.

22. Larsen FJ, Schiffer TA, Ørtenblad N, Zinner C, MoralesAlamo D, Willis SJ, Calbet JA, Holmberg HC and Boushel R. High-intensity sprint training inhibits mitochondrial respiration through aconitase inactivation. FASEB J. 2016; 30: 417-427.

23. Austin CM, Wang G and Maier RJ. Aconitase Functions as a Pleiotropic Posttranscriptional Regulator in Helicobacter pylori. J Bacteriol. 2015; 197: 3076-3086.

24. Maejima Y, Kuroda J, Matsushima S, Ago T and Sadoshima J. Regulation of myocardial growth and death by NADPH oxidase. J Mol Cell Cardiol. 2011; 50:408-416.

25. Lalève A, Vallières $\mathrm{C}$, Golinelli-Cohen MP, Bouton $\mathrm{C}$, Song Z, Pawlik G, Tindall SM, Avery SV, Clain J and Meunier B. The antimalarial drug primaquine targets Fe-S cluster proteins and yeast respiratory growth. Redox Biol. 2016; 7: 21-29.

26. Kim YW and Byzova TV. Oxidative stress in angiogenesis and vascular disease. Blood. 2014; 123: 625-631.

27. Wang Y, Zang QS, Liu Z, Wu Q, Maass D, Dulan G, Shaul PW, Melito L, Frantz DE, Kilgore JA, Williams NS, Terada LS and Nwariaku FE. Regulation of VEGF-induced endothelial cell migration by mitochondrial reactive oxygen species. Am J Physiol Cell Physiol. 2011; 301: C695-704.

28. Sondheimer HM, Lung MC, Brugman SM, Ikle DN, Fan LL and White CW. Pulmonary vascular disorders masquerading as interstitial lung disease. Pediatr Pulmonol. 1995; 20: 284-288.

29. Gao J, Qiu X, Wang X, Peng C and Zheng F. Associations of ChREBP and Global DNA Methylation with Genetic and Environmental Factors in Chinese Healthy Adults. PLoS One. 2016; 11: e0157128.

30. Liu H, Li F, Zhu Y, Li T, Huang H, Lin T, Hu Y, Qi X, Yu $\mathrm{J}$ and $\mathrm{Li} \mathrm{G}$. Whole-exome sequencing to identify somatic mutations in peritoneal metastatic gastric adenocarcinoma: A preliminary study. Oncotarget. 2016; 7: 43894-43906. doi: 10.18632/oncotarget.9707.

31. Gotardo ÉM, Caria CR, de Oliveira CC, Rocha T, Ribeiro ML, Gambero A. Effects of iron supplementation in mice with hypoferremia induced by obesity. Exp Biol Med 
(Maywood). 2016; doi: 10.1177/1535370216660398. [Epub ahead of print].

32. Astekar M, Joshi A, Ramesh G and Metgud R. Expression of vascular endothelial growth factor and microvessel density in oral tumorigenesis. J Oral Maxillofac Pathol. 2012; 16: 22-26.

33. Chen D, Li L, Tu X, Yin Z and Wang Q. Functional characterization of Klippel- Trenaunay syndrome gene
AGGF1 identifies a novel angiogenic signaling pathway for specification of vein differentiation and angiogenesis during embryogenesis. Hum Mol Genet. 2013; 22: 963-976.

34. Ott de Bruin L, Yang W, Capuder K, Lee YN, Antolini M, Meyers R, Gellert M, Musunuru K, Manis J and Notarangelo L. Rapid generation of novel models of RAG1 deficiency by CRISPR/Cas9-induced mutagenesis in murine zygotes. Oncotarget. 2016;7:12962-12974. doi: 10.18632/ oncotarget. 7341 . 